\title{
Felling of Individual Freestanding Nanoobjects Using Focused-ion-beam Milling for Investigations of Structural and Transport Properties
}

\author{
Wuxia Li, ${ }^{1.2}$ J.C. Fenton, ${ }^{2}$ Ajuan Cui, ${ }^{1}$ Huan Wang, ${ }^{2}$ Yiqian Wang, ${ }^{3}$ Changzhi Gu, ${ }^{1}$ D.W. McComb, ${ }^{3}$ \\ and P.A. Warburton ${ }^{2}$ \\ ${ }^{1}$ Beijing National Lab of Condensed Matter Physics, Institute of Physics, Chinese Academy of \\ Sciences, Beijing 100190, China \\ ${ }^{2}$ London Centre for Nanotechnology, University College London, London, WC1E 7JE, UK \\ ${ }^{3}$ London Centre for Nanotechnology, Imperial College, London, SW7 2AZ, UK \\ Email: liwuxia@aphy.iphy.ac.cn
}

Short title: Felling of freestanding objects for properties investigation

PACS: 81.16.-c, 87.85.Rs, 81.07.-b

\begin{abstract}
We report that, to enable studies of their compositional, structural and electrical properties, freestanding individual nanoobjects can be selectively felled in a controllable way by the technique of low-current focused-ion-beam (FIB) milling with the ion beam at a chosen angle of incidence to the nanoobject. To demonstrate the suitability of the technique, we report results zigzag/straight tungsten nanowires grown vertically on support substrates and then felled for characterization. We also describe a systematic investigation of the effect of the experimental geometry and parameters on the felling process and on the induced wire-bending phenomenon. The method of felling freestanding nanoobjects using FIB is an advantageous new technique for enabling investigations of the properties of selected individual nanoobjects.
\end{abstract}

\section{Introduction}

Recently, with the downscaling of electronics, nanomaterials with various shapes have been synthesized and their properties have been explored by several different methods [1-7], with a view to building novel nanodevices and new functional logic circuit architectures. Of critical importance for applications is the ability to measure the properties of an individual freestanding nanoobject, to provide feedback for improving their properties to obtain the nanomaterials desired for constructing high-density three-dimensional circuits [8-11]. However, depending on the shape of the nanoobject and the distribution of groups of nanoobjects, the characterization of structural, compositional and electrical properties may not be possible for some characterization techniques.

Up to now, for the characterization of the properties of nanoobjects, the most popular method has been to use a blade to scratch the support substrate and release the nanomaterials, sonicate in ethanol for dispersion and then transfer to: either (i) a transmission electron microscope grid for structural and compositional analysis [12]; or (ii) a substrate covered with a layer of dielectric material such as $\mathrm{SiO}_{2}$ for characterization of electrical and optical properties [13]. To form nanocontacts on individual nanomaterials after scratching and redistribution on the substrate surface for transport properties characterization, very recently, focused-ion-beam induced chemical vapour deposition (FIB-CVD) has been employed for this purpose extensively [14-17]. The main abilities of FIB technique include the removal and deposition of materials with a high precision, which provides the ideal tool for the rapid prototyping of a whole range of devices in areas such as organic semiconducting nanodevices [14], microelectro-mechanical systems [15], superconducting devices [16] and optical applications [17]. In particular, FIB-CVD allows the formation of complex interconnects in a user-defined area in a single processing step, with resolution comparable to the 
structures defined by electron-beam lithography. However, the disadvantages of the scratch-pickdispersion-transfer technique include: (i) mechanical damage affecting the shape of the nanomaterials and stress induced by blade scratching, especially for limbed and complex nanostructures; (ii) it is time-consuming and difficult to find a suitable nanoobject (sufficiently long and with a substantial distance from the neighboring parts) for contact fabrication; (iii) it is extremely difficult or impossible to find the particular nanoobject selected before scratching.

Thus a precise and controllable technique for releasing a freestanding nanoobject from the support substrate is clearly needed. In this letter, we describe a technique we have developed for felling freestanding nanowires by means of low-current focused-ion-beam (FIB) milling. To demonstrate this, we have grown vertical zigzag tungsten nanowires on holey carbon grids and then felled them for structure and composition analysis. We also present results on vertical zigzag/straight tungsten nanowires grown on a $\mathrm{Si}$ substrate which was coated with a layer of $200 \mathrm{~nm} \mathrm{SiO}_{2}$. We present the temperature-dependent resistance and low-temperature current-voltage dependence after the freestanding grown nanowires were felled and we describe the effects of experimental geometry on the felling process and on the phenomenon of ion-beam-induced bending. Our results demonstrate that felling of freestanding nanoobjects by FIB milling is an effective method for investigating the properties of particularly interesting individual nanoobjects, provided the areal density of these objects is not too high. ${ }^{1}$

\section{Experimental system}

A commercially available SEM/FIB system utilizing a beam of $30 \mathrm{keV}$ singly charged $\mathrm{Ga}^{+}$ ions was used to grow and fell tungsten composite nanowires. The system was equipped with a gas injection module for ion-beam induced deposition. The $\mathrm{W}(\mathrm{CO})_{6}$ gas was introduced to the substrates through a nozzle, creating a local high pressure in the region scanned by the ion-beam without substantial pressure rise in the rest of the work chamber. The base pressure before introducing the precursor gas was $2.4 \times 10^{-6}$ mbar and during deposition the pressure was in the range $7.4-8.3 \times 10^{-6}$ mbar.

Deposition can be induced by irradiating one point on the substrate using spot mode. In that case, a straight wire is grown. Alternatively, the ion-beam may be raster-scanned repeatedly over a rectangular area, first across the area in one direction and then back in the other direction; if the scan speed in one of the dimensions across the wire is slow, then a zigzag profile results. An example of a wire resulting from this growth method shown by the TEM bright field and scanning transmission electron microscope (STEM) images in Fig.1 (a) and (b) respectively.
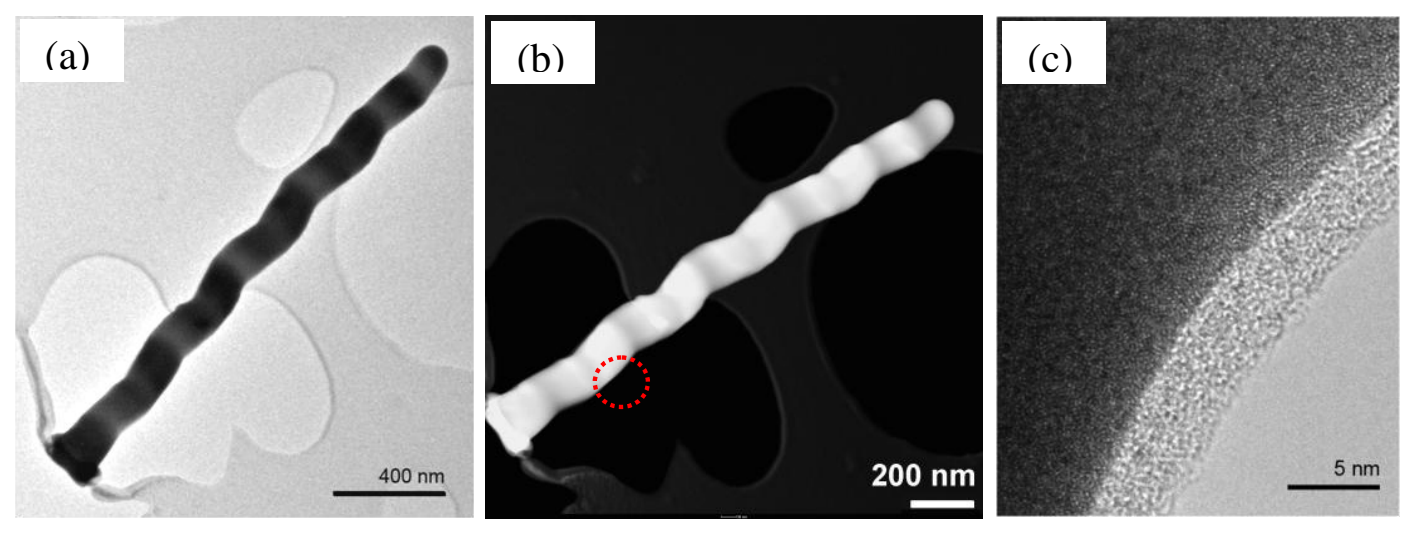

\footnotetext{
${ }^{1}$ In some cases, for instance, to study the electrical properties of an individual nanowire grown by chemical vapor deposition on conducting substrate (usually a forest of nanowires are grown randomly), scratching and redistribution of nanoobjects rather than FIB felling is the more suitable method.
} 
Figure 1. TEM images of a freestanding tungsten nanowire after being felled by FIB milling: (a) Bright field TEM image; (b) STEM image; (c) HRTEM image of the area of the wire indicated by the dashed lines in Fig. 1(b).

The experimental geometry offers flexibility for controlling both the cutting angle and the cutting position along the wire length. Although the ion beam direction in FIB systems is generally fixed, in order to fell nanoobjects the angle of the ion-beam with respect to the nanoobject may be modified by tilting the sample stage. Different possible arrangements are shown in Fig. 2. In our system, the ion beam is at an angle of $54^{\circ}$ to the vertical, so the stage may be oriented with the ion beam perpendicular to the substrate surface for vertical nanowire growth (as in Fig. 2a) by titling the stage by $54^{\circ}$ or parallel to the substrate surface (as in Fig. 2d) by employing a tilted sample holder with a facet tilt angle of $45^{\circ}$ and tilting the stage by $9^{\circ}$. Alternatively, felling may be carried out with the stage tilted at a different angle; for example, a stage-tilt angle of $32^{\circ}$ leads to an angle between the incident beam and the wire of $20^{\circ}$ (as in Fig. 2b). The maximum relative angle between the incident ion beam and the vertically grown nanoobject is $90^{\circ}$ since at larger angles the ion beam is blocked by the upper edge of the substrate. Fig. 3 shows SEM images of one straight tungsten nanowire before (a), during (b) and after (c) felling.

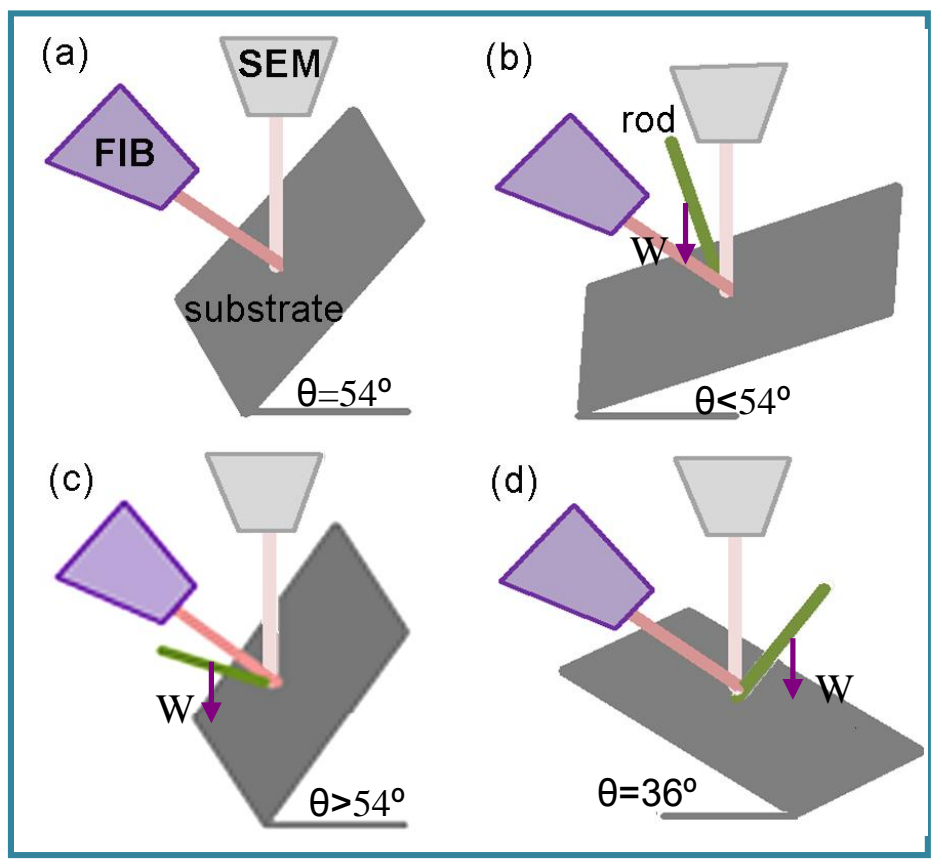

Figure 2. Schematics of the experimental geometry illustrating the relative position of the incident ion beam and electron beam, the freestanding nanoobject, the direction on which the object's weight (W) acts from its centre-of-gravity and the substrate: (a) ion-beam normal to substrate for vertical nanorod growth, the tilt angle $(\theta)$ of the substrate is $54^{\circ}$ from horizontal; (b) $\theta<54^{\circ}$ and (c) $\theta>54^{\circ}$ ion-beam at a general angle for cutting, the nanorod's weight (W) acting from its centre-of-gravity may act towards (b) or in the opposite direction (c) from the incident ion beam. Note that the angle between the nanorod and the incident ion beam may have positive or negative sign; (d) ion-beam normal to wire - oblique incidence for cutting, $\theta=-36^{\circ}$ by using a sample holder with a tilt facet of $45^{\circ}$, in such a geometry the cutting area is minimized and milling of the substrate can be virtually avoided.

\section{Results and discussions}

\subsection{Felling of freestanding nanowires for structural and compositional studies}


To demonstrate the suitability of the FIB felling technique for examining the structural and compositional properties of (previously) freestanding nanomaterials, tungsten-containing vertical zigzag nanowires were grown on a holey carbon transmission electron microscope (TEM) grid by FIB-induced chemical vapour deposition.

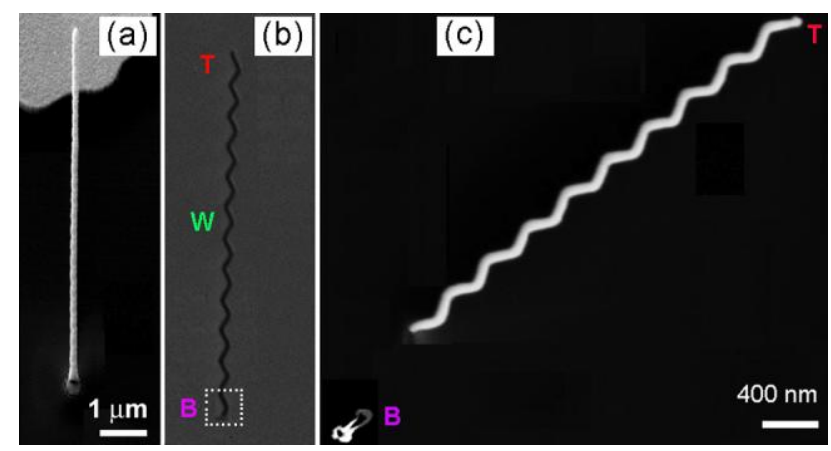

Figure 3. Images of vertically grown tungsten nanowires illustrating the felling process using a $1 \mathrm{pA}$ ion beam normally incident to the wire length direction: (a) is an SEM image of the as-deposited nanowire with a viewing angle of $54^{\circ}$ (the zigzags are in a plane parallel to the viewing direction and so are not visible); (b) is an FIB image of the freestanding nanowire shown in (a) with the ion beam normal to its length and parallel to the support-substrate surface; (c) the nanowire has been felled away from the incident ion beam, and is lying on the substrate surface close to its base, with the top downwards.
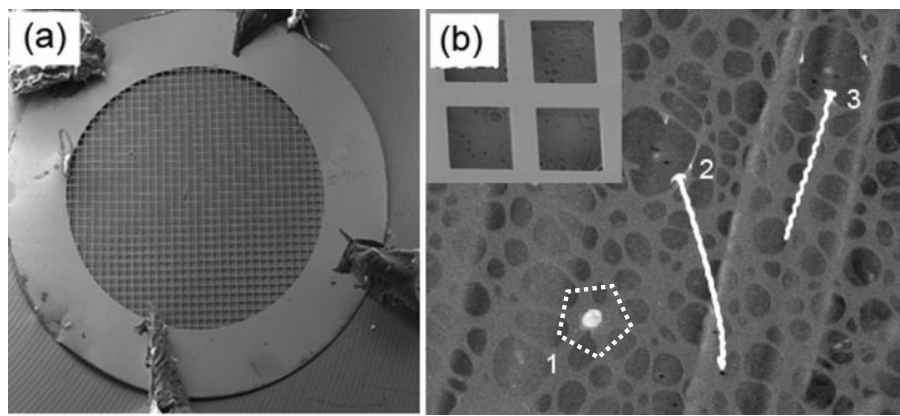

Figure 4. (a) Transmission electron microscope (TEM) grid fixed on a scanning electron microscope (SEM) sample holder; (b) SEM top-view image of freestanding tungsten composite wires: an asdeposited wire (1), wires lying on the TEM grid felled by FIB ((2) and (3)). The inset shows the square grid that supports the holey carbon film; the dotted line schematically shows the cutting position of carbon network that support the as-deposited wires for felling.

Figure 4 (a) shows a plan-view SEM image of a holey carbon TEM grid attached onto a SEM sample holder by conductive carbon tape stuck from the topside of the grid to the sample holder. The tape fixed the TEM grid and provided a charge-transfer channel. More importantly, it was easy to peel off the carbon tape from the top after felling and thus protect the grid, which was to be checked by TEM afterwards. The rods were grown with the beam normal to the substrate surface by scanning the beam in an area $100 \mathrm{~nm} \times 100 \mathrm{~nm}$, using $1 \mathrm{pA}$ ion beam current. To fell the nanowires, interlinks of the supporting carbon film in the vicinity of a freestanding nanowire were cut through with a $1 \mathrm{pA}$ ion beam normal to the membrane surface. Fig.4 (b) shows an SEM image of the felled tungsten nanowires lying on the holey carbon grid; the top view of the as-grown nanowire is also indicated. The dotted white line schematically shows the FIB cutting positions of the supporting carbon networks. A 1 pA ion beam current was used to cut the interlinks that support the nanowire. Upon losing its support, the wire was felled and lay on the holey carbon grid as shown in Fig.4 (b).

The composition and structure were examined by electron-energy loss and energy dispersive $\mathrm{x}$-ray spectroscopy, scanning electron microscope and high-resolution transmission electron microscope. A high-resolution TEM image is shown in Fig. 1 (c). This shows that the nanowires do not display any long-range order - rather there are nanocrystallites with grain size on the order of 1 
$\mathrm{nm}$. Electron-energy loss and energy dispersive $\mathrm{x}$-ray spectroscopy show that the composition is 48 at $\%$ tungsten, 30 at $\%$ carbon, 16 at $\%$ gallium and 6 at $\%$ oxygen. This composition is similar to that reported previously [18-20], indicating that FIB felling of the as-deposited objects leads to no changes in their structural properties. (Note also that this shows that FIB-deposited tungsten nanowires have different composition and structure from bulk crystalline tungsten and electron beam deposited tungsten composite.)

\subsection{Felling of freestanding nanowires grown on $\mathrm{SiO}_{2} / \mathrm{Si}$ substrates for transport investigations}

For measurements of transport properties, patterns for the electrical contacts can be fabricated by using electron-beam lithography and/or photolithography [13] onto a selected individual nanowire and then metallic layers can be deposited by electron beam evaporation, thermal evaporation or sputtering, followed by lift-off. FIB-induced deposition of Pt [7, 21] and W [7] composites can be used to direct-write electrical connection and contact pads on individual nanowires. By this technique, patterns with resolution comparable to those made by electron beam lithography can be made in a single step, thus avoiding resist patterning and metal deposition processes.

To demonstrate the felling of freestanding nanoobjects for analysis of their electrical properties, straight and zigzag tungsten nanowires were vertically grown on 200-nm-thick gold test patterns previously deposited on a 200-nm-thick $\mathrm{SiO}_{2}$ layer on a $\mathrm{Si}$ substrate using conventional microelectronic techniques. Fig. 3 (a) is an SEM image of the vertically grown zigzag nanowire with a viewing angle of $54^{\circ}$. Fig. 3 (b) is an FIB image of the freestanding nanowire as shown in Fig. 3 (a) with the ion beam normal to its length (see Fig. 2d). To fell the wire, a 1 pA focused ion beam was first used to scan an area of $400 \mathrm{~nm} \times 400 \mathrm{~nm}$ near the base of the nanowire for 2 minutes, as shown by the dotted white rectangle. It can be seen from the SEM plan-view image shown in Fig.3 (c) that the wire was felled with the tip away from the incident ion beam (indicated by letter $\mathrm{T}$ ), that the wire landed less than $2 \mu \mathrm{m}$ from its base (indicated by letter B) and that the shape and size of the wire are unchanged.

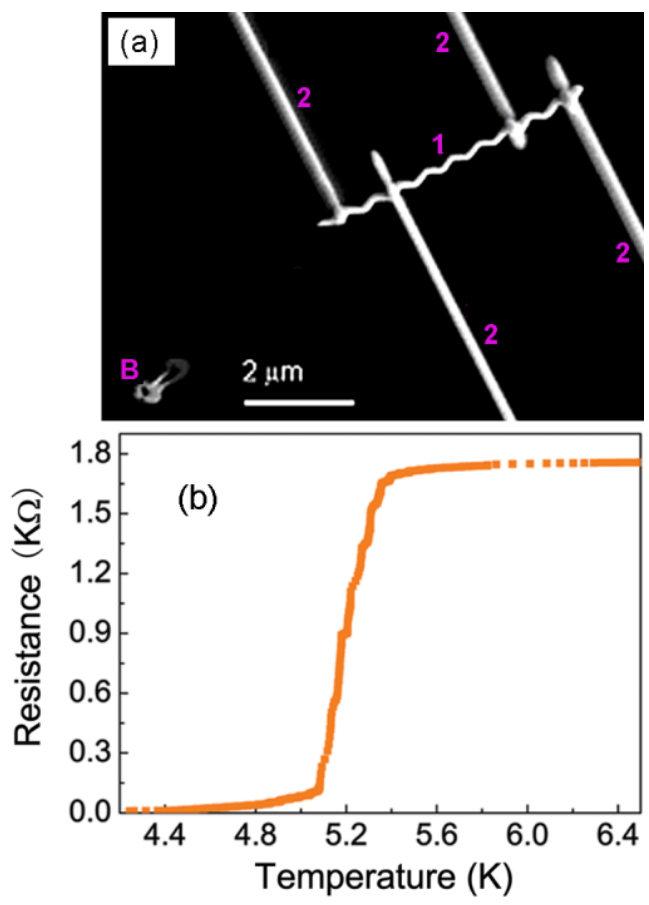

Figure 5. (a) SEM top-view of a typical four-terminal configuration based on a felled nanowire showing (1) the felled wire and (2) the tungsten connectors grown by FIB induced deposition; (b) the temperature-dependent resistance of the felled nanowire; the inset shows the low temperature region.

After felling onto the substrate surface, FIB-induced deposition was used to fabricate contact pads and connectors for the measurement of electrical properties. Figure 5 (a) shows a typical such structure with a four-terminal configuration. The base after cutting is indicated by letter B; the numbers show the felled wire (1) and the tungsten connector (2) deposited in-situ by using an ion 
beam current of $30 \mathrm{pA}$. It can be seen that the wire lies very close to its base; the top of the wire pointing is downwards. Low-temperature transport measurements were performed with a helium dip-probe and home-made current source and voltage amplifiers. A constant current of $1.0 \mu \mathrm{A}$ was applied to the two outer terminals and the voltage was measured between the other two terminals. A temperature-dependent resistance measurement on a felled nanowire is shown in Fig. 5 (b). In the inset is the R-T curve for the low temperature region, from which the superconducting transition temperature $\left(T_{c}\right)$ was derived to be of $5.2 \mathrm{~K}$. Here $T_{c}$ is defined as the temperature at which the resistance falls to $50 \%$ of its value at the onset of the transition. The room-temperature resistivity of the zigzag nanowire was calculated to be about $520 \mu \Omega \mathrm{cm}$, a value similar to that of an air-bridge structure also grown by $1 \mathrm{pA}$ ion beam current [22]. The results reveal that felling freestanding nanowires using $1 \mathrm{pA}$ ion beam current does not affect their intrinsic electrical properties. For comparison, bulk tungsten has a resistivity of $5 \mu \Omega \mathrm{cm}$ at room temperature. Resistivity values from about $100 \mu \Omega \mathrm{cm}$ to $300 \mu \Omega \mathrm{cm}$ have been reported for tungsten composites grown by FIB-induced deposition $[18,23,24]$. The variation in the resistivity of FIB-deposited materials is a consequence of the microstructure and composition difference due to the operating parameters, such as the ionbeam current, dose, substrate material, substrate temperature, scan speed and gas flux.

To further understand the electrical properties in FIB deposited nanowires, current-voltage measurements were performed at $4.26 \mathrm{~K}$. The zero-field I-V curve is shown in Fig. 6. This shows a critical current of $23 \mu \mathrm{A}$ and thus these results demonstrate the use of FIB-CVD to direct-write superconducting nanostructures. Distinct linear branches can also be seen when the current is reduced. These features of the I-V curve suggest the occurrence of thermally activated phase slip processes as they are strongly reminiscent of the I-V characteristics of phase-slip centres [25, 26]. A phase slip results from the fluctuation of the superconducting order parameter at some point along the wire and leads to a voltage pulse; this means that a thin superconducting wire below $\mathrm{T}_{\mathrm{c}}$ can show finite electrical resistance.

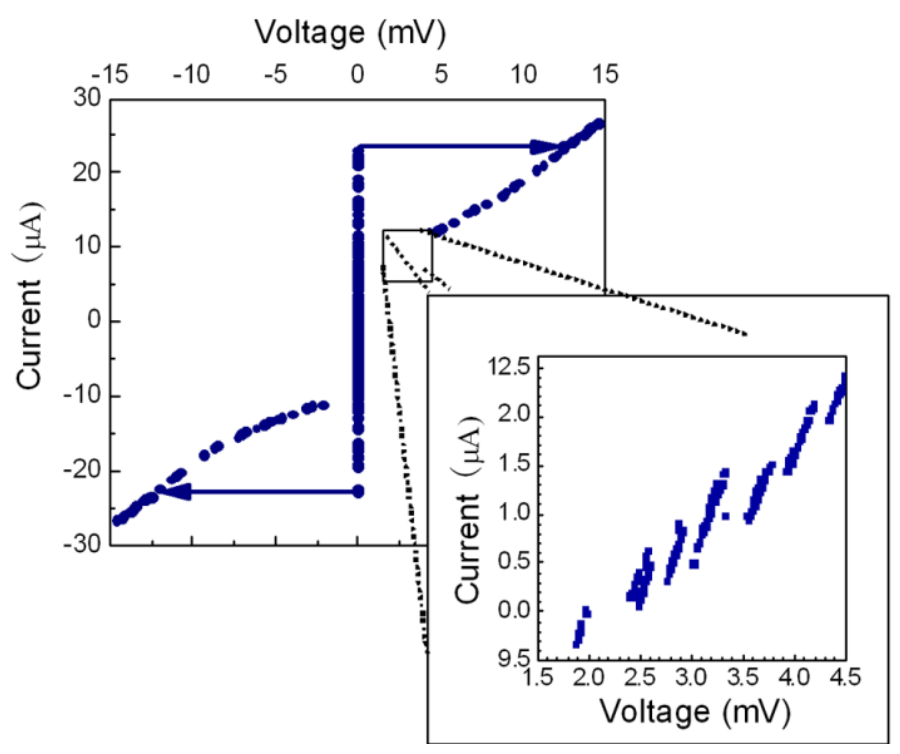

Figure 6. Zero-field I-V characteristics at $4.26 \mathrm{~K}$ for the felled tungsten nanowire shown in Fig.5 (a).

\subsection{Wire felling direction}

To investigate ion-beam-induced felling in our experimental system in more detail, we measured more than 100 nanowires under different conditions. Unless beam-induced bending of the wire is significant (discussed below, in Section 6), the felling direction (towards or away from the incident ion beam) is mainly governed by the relative position of the incident ion beam and the centre-of-mass of the nanowire.

For a particular freestanding nanoobject, the felling direction is closely related to the geometry used for felling. The geometries used to grow and fell the nanowires are illustrated in Fig. 2. Fig. 2 (a) shows the geometry used for vertical nanowire growth with the beam normal incident to the substrate. We found that under conditions with the beam incident obliquely on the nanowire (- 
$36^{\circ}<\theta<62^{\circ}$ ), as shown in Fig. 2 (b), (c) and (d), using raster scans over an area of order $2 \mu \mathrm{m} \times 0.5 \mu$ $\mathrm{m}$, with $1 \mathrm{pA}$ beam current, the wires were most often felled with the tip pointing to the lower part of the substrate, i.e. in the same direction as the moment due to the nanoobject's weight - "downhill"; this could be either towards (Fig. 2(b)) or away from (Fig. 2(c) and Fig. 2(d)) the incident ion beam because varying the tilt angle of the substrate as shown in Fig. 2(b)-(d) changes both the angle at which the ion beam is incident on the freestanding nanoobject and the moment due to the object's weight (W) about its base.

One advantage of felling at normal incidence (Fig. 2d) is that a minimum cutting crosssection is obtained when the ion beam used for felling is normal to the wire length. This geometry minimizes the milling time and also minimizes ion irradiation of the substrate, avoiding possible damage to the top dielectric layer of the substrate. For almost-normal incidence, if felling was finished by one run of cutting with $1 \mathrm{pA}$ and using a rectangle area smaller than $2 \mu \mathrm{m} \times 0.5 \mu \mathrm{m}$, the nanowire was always felled downhill. For nanowires not cut through by one run of felling, imaging (especially using large (e.g. 50pA) ion beam current) can also fell the wire, towards the incident ion beam direction. We discovered that there is also some dependence of the felling direction on the details of the felling. When felling was carried out at normal incidence (Fig. 2d) with a larger 50pA beam current using a single-scan over a smaller area, the nanowires were always observed to fall towards the beam.

In general, the landing position of the felled wire on the substrate is cutting-position- and tiltangle-dependent. The further the beam scanning area from the base of the wire, the more distant the landing position; however, on some occasions when wires were cut higher up from the base, they would tend to fall over but remain attached at the cutting position. This leads to the practical conclusion that the selected nanowire should be felled near its base. Although the felling might be towards or away from the direction of the incident ion beam, the landing position of the felled object in most cases is then close to its base. This is then easy to locate for electrode fabrication.

\subsection{Investigation of ion-induced wire bending}

Irradiation of nanomaterials and surfaces with energetic ions can result in either unwanted or desired changes of the morphology due to several different atomistic effects [27-30]. The most wellknown effect is that of sputtering - the removal of the irradiated surface atoms when the impact energy of the incident ions transferred to them. The felling of freestanding nanoobjects such as described above is based on the sputtering effect. Another very recently reported effect caused by the beam-matter interaction is the bending and alignment of suspended nanoobjects with ion irradiation [31,32]. Fig. 7 (a) shows an example of vertical grown nanowires, as-grown (Fig. 7 (a1) and bending after irradiation with a $30 \mathrm{pA}$ ion beam (Fig. 7 (a2)-(a4). This is caused by ion-beam-induced plastic deformation, a very complex behavior governed by many factors including: (i) the geometry, size, thermal expansion coefficient, Young's modulus and crystalline properties of the freestanding nanoobjects; (ii) the electrical and thermal conductivity of the support substrate; and (iii) the ion irradiation conditions, which include the ion beam energy, the incident angle, the ion beam current and the ion beam scanning strategy [25,26, 31-32]. All these factors compete against or combine with each other to cause the freestanding nanoobject to bend away from or towards the incident ion beam. 


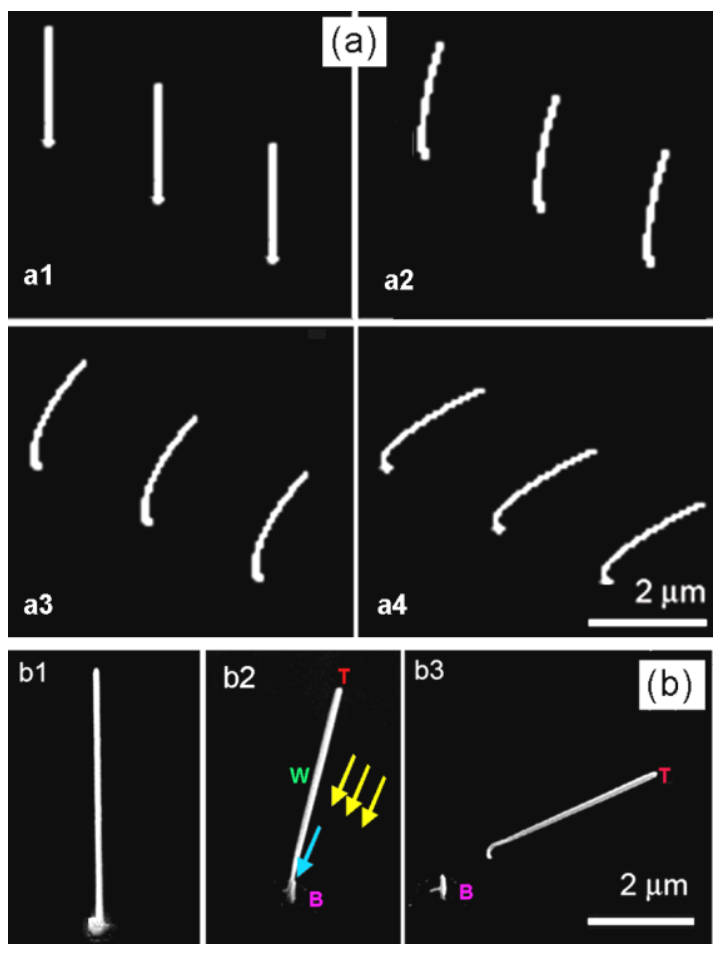

Figure 7. Images of vertically grown straight tungsten nanowires illustrating the bending and felling processes (a) ion-induced bending of nanowire irradiated with a $30 \mathrm{pA}$ ion beam oblique incidence to the wire length direction $\left(\theta=60^{\circ}\right)$ : (a1) is an SEM image of the as-deposited nanowire with a viewing angle of 54\%; (a2)-(a4) shows the SEM image of nanowire irradiated with ion dose of 22 $\mathrm{nC} / \mu \mathrm{m}^{2}, 66 \mathrm{nC} / \mu \mathrm{m}^{2}$ and $110 \mathrm{nC} / \mu \mathrm{m}^{2}$ respectively; (b) nanowire felled with $30 \mathrm{pA}$ ion beam at oblique incidence to the wire length direction $\left(\theta=60^{\circ}\right)$ : (b1) is an SEM image of the as-deposited nanowire with a viewing angle of $54^{\circ}$; (b2) is an SEM image of the freestanding nanowire after being cut at the base for $30 \mathrm{~s}$; (b3) nanowire been felled towards the incident ion beam with the top upwards lying on the substrate surface close to its base. W, T and B denote respectively the centreof-mass, the tip and the base of nanowire, the yellow arrow shows the beam direction and the blue arrow shows the FIB cutting position.

In our systematic study of over 100 nanowires, we have observed that there is some dependence of the bending behaviour on the substrate. Tungsten and platinum nanowires were grown on isolated $\mathrm{Au}$ pads on $\mathrm{SiO}_{2} / \mathrm{Si}$ substrates or directly onto $\mathrm{Si}$ or $\mathrm{Al}$ or $\mathrm{Au}$ substrates and then irradiated with oblique incidence $\left(0^{\circ} \leq \theta \leq 60^{\circ}\right)$ using ion beam current ranging from $10-100 \mathrm{pA}$, using a magnification of 12,000 and using a raster scan, with one sweep of the field of view taking $162 \mathrm{~s}$. The wires grown on the isolated $\mathrm{Au}$ pads were observed to bend first away from the ion beam then towards the ion beam, whereas the wires grown directly on the substrate always bent towards the incident ion beam. More details and the mechanism behind such phenomenon will be reported elsewhere.

Nanowires were also imaged using a $1 \mathrm{pA}$ ion beam. For nanowires shorter than $7 \mu \mathrm{m}$ in length and larger than $100 \mathrm{~nm}$ in diameter, no bending was observed for nanowires grown on any of the substrates used, even when the whole nanowire had received an ion dose ten times larger than required for felling. This was the case both for normal incidence and in the oblique-incidence geometry $\left(0^{\circ} \leq \theta \leq 60^{\circ}\right)$. For nanowires having both high aspect ratio e.g. 90 and being imaged under high magnification, e.g. 50,000, bending was again observed. After being partially cut at the base, scanning could again cause the nanowire to bend either towards or away from the incident ion beam. For nanowires with diameter smaller than $100 \mathrm{~nm}$, no bending was observed with $1 \mathrm{pA}$ irradiation when a magnification less than 15,000 was used. When higher magnification, e.g. 80,000 was used, these small-diameter nanowires were also observed to bend under 1 pA irradiation. 
This variety of behaviours as the parameters are changed further indicates that the bending is a complicated phenomenon that is governed by many factors. Since it is generally worthwhile to avoid imaging-related bending during felling by using low ion-beam current, our practical conclusion is that, to avoid bending during imaging, $1 \mathrm{pA}$ ion beam current may be chosen to take an image.

In general, it is the combined effect of bending and gravity that determines the felling direction of the nanowire, since the bending direction is a complicated phenomenon that is controlled by many factors. The centre-of-mass of the irradiated nanowire dynamically changes with the evolution of the shape of the nanoobject during bending, so the nanoobject could be felled either towards or away from the incident ion beam with its tip pointed upwards or downwards, depending on the relative strength of the moment due to the object's weight and that resulting from the bending effect caused by ion beam irradiation during imaging and cutting.

The bending effect could also affect the felling process and the final felling direction of the nanoobject under investigation. Figure 7(a) demonstrates the effect that bending can have on the milling process. An SEM image of a nanowire before milling is shown in Fig. 7(b). To fell the nanowire, an FIB image was first grabbed using a frame scanning time of 60s, then an area of 400 $\mathrm{nm} \times 400 \mathrm{~nm}$ near the base of the nanowire (as shown by the dotted white rectangle) was scanned with $30 \mathrm{pA}$ for $30 \mathrm{~s}$. An SEM image was taken at this point; this is shown in Fig. 7 (b2). After this, another FIB image was taken and this was used as the reference image for further milling for 1 minute. An SEM image after this further milling, Fig.6 (b3), shows that the nanowire has been felled.

It can be seen from Fig. 7 (b2) that after FIB scanning with $30 \mathrm{pA}$ ion beam current, the wire bent towards the incident ion beam with the cutting point at the base being thinned. Further imaging and cutting caused the tip of the nanowire finally to point to the higher part of the substrate, which shows that the up-dragging moment induced by the ion beam induced bending of the whole nanowire has overtaken that imposed by the down-pulling effect of the weight of the nanowire.

\section{Conclusion}

In summary, we have demonstrated that low-current FIB milling may be used to fell freestanding nanoobjects for compositional, structural and electrical properties investigations. The TEM results and electrical properties analysis show that, firstly, the felling process exhibits negligible side effects on the structures of nanowires; secondly, the technique provides a particularly flexible, controllable and target-specific approach for the exploration of the properties of freestanding nanoobjects; thirdly, the current-voltage (I-V) characteristics of the felled nanowire show a series of discrete steps in approaching the normal state, indicating that FIB-induced deposition of vertical nanowires might be a potential approach to probe the quantum phenomena in nanoscale tungsten with significantly enhanced superconductivity - this opens up the possibility of fabricating novel superconducting devices without a mask or other conventional micro-fabrication techniques. By investigating a large number of nanowires, we have demonstrated that the phenomenon of bending is a complex process dependent on many factors. For felling nanowires for transport investigations, a $1 \mathrm{pA}$ beam scanned close to the base of the nanowire is recommended.

\section{Acknowledgment}

The authors acknowledge Kevin Lee for technical assistance, O. Chiatti for discussion. This work is supported by National Natural Science Foundation of China under Grant No.91123004, 11104334, 50825206, Outstanding Technical Talent Program of Chinese Academy of Sciences, IRC in Nanotechnology, and EPSRC contract EP/F035411/1

\section{References}

[1] Katsaros G. Spathis P, Stoffel M, Fournel F, Mongillo M, Bouchiat V, Lefloch F, Rastelli A, Schmidt O G and Franceschi S De Nature Nanotechnology 2010 DOI: 10.1038.

[2] Kim J R, Kim B K, OLee J, Kim J, Seo H J, Lee C J and Kim J J Nanotechnology 200415 1397.

[3] Qu Y Q, Liao L, Li Y J, Zhang H Huang and Duan X F Nano Lett 200994539. 
[4] Bruhn B, Valenta J, Linnros J Nanotechnology 200920505301.

[5] Lai C X, Wu Q B, Chen J, Wen L S and Ren S Nanotechnology 201021215602.

[6] Qu Y Q, Liao L, Cheng R, Wang Y, Lin Y C, Huang Y and Duan X F Nano Lett. 2010 DOI:

10.1021.

[7] Newton M C, Firth S and Warburton P A Appl. Phys. Lett. 200689072194.

[8] Guo X, Qiu M, Bao J M, Wiley B J, Yang Q, Zhang X N, Ma Y G and Tongk L M Nano Lett. 200994515.

[9] Magasinski A, Dixon P, Hertzberg B, Kvi A, Ayala J and Yushin G Nature Materials 20109353. [10] Assefa S, Xia F N and Vlasov Y A Nature 201046480.

[11] Xu S, Qin Y, Xu C, Wei Y G, Yang Rm and Wang Z L Nature Nanotechnology 2010 DOI: 10.1038.

[12] He Y, Wang J A, Chen X B, Zhang W F, Zeng X Y, Gu Q W, J. Nanopart. Res. 201012169.

[13] Long R H, Chen J J, Lim J H, Bwiley J and Zhou W L, Nanotechnology $200920285306 .$.

[14] Long Y Z, Duvail J L, Li M M Gu C Z, Liu Z W and Ringer S P Nanoscale Res Lett 20105 237.

[15 ] Li Y J, Xie H M, Guo B Q, Luo Q, Gu C Z and Xu M Q J Micromech. Microeng. 201020 055037.

[16] Romans E J, Osley E J, Young L, Warburton P A, Li W Appl. Phys. Lett. 2010 97, 222506.

[17] Temnov V V, Armelles G, Woggon U, Guzatov D, Cebollada A, Garcia-Martin A, GarciaMartin G M, Thomay T, Leitenstorfer A,and Bratschitsch A Nature Photonics 20104107.

[18] Ross I M, Luxmoor, I J, Gullis A G, Orr J, Buckle P D, Jefferson J H Journal of Physics: Conference series 2006, 26, 363.

[19] Li W, Fenton C J, Wang Y, McComb DW and Warburton P A J. Appl. Phys. 2008 104, 093913. [20] Li W, Fenton J C and Warburton P A IEEE Trans. Appl. Supercond. 2009192819.

[21] Lu S L, Song Z T and Liu Y, Feng S L Chia . Phys. Lett. 201027028401.

[22] Li W and Warburton P A Nanotechnology 200718485305.

[23] Prestigiacomo M, Bedu, Jandard F, Tonneau D, Dallaporta H, Roussel L and Sudraud P Appl. Phys. Lett. 200586192112.

[24] Nakamatsu K, Igaki J, Nagase M, Ichihashi T, Matsui S, Microelectronic Engineering 200683 808.

[25] Tian M, Wang J, Kurtz J S, Liu Y, Chan M H W, Mayer T S and Mallouk T E Phys. Rew. B 200571104521.

[26] Lau N, Markovic N, Bockrath M, Bezryadin A and Tinkham M Phys. Rev. Lett. 200187 217003.

[27 Lim Y C, Westerwalbesloh T, Aschentrup A, Wehmeyer O, Haindl G, Kleineberg U and Heinzmann U Appl. Phys. A 200172121.

[28] Hedler A, Klaumunzer S L and Wesch W Nat. Mater. 20043804.

[29] Snoeks E, Blaaderen A, Dillen T, Kats C. M, Brongersma M L and Polman A Adv. Mater. 2000121511.

[30] Chan W L, Chason E and Iamsumang I Nucl. Instrum. Methods Phys. Res. Sect. B 2007257 428.

[31] Borschel C, Niepelt R, Geburt S, Gutsche C, Regolin I, Prost W, Tegude F-J, Stichtenoth D, Schwen D and Ronning C 2009 Small 5 2576-2580.

[32] Borschel C, Spindler S, Lerose D, Bochmann A, Christiansen S H, Nietzsche S, Oertel M AND Ronning C Nanotechnology 201122185307. 